An official publication of the International Society for Energy, Environment and Sustainability (ISEES)

Journal of Energy and Environmental Sustainability

Journal homepage : www.jees.in

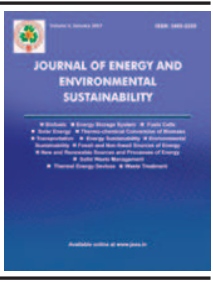

\title{
Estimation of Interfacial Evaporative Heat Flux using Inverse Heat Transfer Framework for Passive Solar Distillation System
}

\author{
Manish Bhendura ${ }^{\mathrm{a}}$, Samarjeet Chanda ${ }^{\mathrm{b}}$ \\ ${ }^{a}$ Department of Mechanical Engineering, IIT Kanpur, Kanpur 208016, UP \\ ${ }^{b}$ Department of Mechanical Engineering, IIT Palakkad, Palakkad 678557, Kerala
}

\section{A R T I C L E I N F O}

Received : 15 July 2019

Revised : 05 September 2019

Accepted : 06 September 2019

Keywords:

Interfacial evaporative heat flux, Surrogate modelling,

Inverse heat transfer framework,

Artificial neural network (ANN),

Genetic algorithm (GA)

\begin{abstract}
A B S T R A C T
In the present study, a methodology is developed to estimate the evaporative heat flux in a solar still mimicking configuration. A distributed heat transfer analysis utilizing the air-water interface temperature instead of bulk temperature of water (lumped analysis) is performed to develop an improved correlation, for predicting interfacial evaporative heat flux. The physical problem is simulated numerically using the method of finite-differences. A surrogate model closely mimicking the physical problem is then developed using artificial neural networks to improve the computational economy. The surrogate model is combined with genetic algorithm in an inverse framework to estimate the parameters of the proposed correlation. Depth of water in the solar still is explicitly included in the correlation to study its effect on the rate of evaporation at the air-water interface. The parameters of the proposed correlation are estimated for a given water depths and initial water temperature using simulated measurements obtained from an ab initio model of simultaneous heat and mass transfer for the considered air-water system.
\end{abstract}

\section{Introduction}

A passive solar distillation system is an eco-friendly and low cost technique to produce potable water in the energy deficit areas [Rajaseenivasanet al., 2017]. It comprises of a basin which is thermally insulated from all sides except the glass cover at the top. The basin is partially filled with water, which is heated by the solar insolation and gets evaporated. Since the glass cover is at a lower temperature, the watervapour condenses over it. This condensate is collected through a suitable drainage arrangement and used as potable water. The productivity of these systems largely depends on the evaporation rate of water which is a strong function of its surface temperature. The partial vapour-pressure of air (moisture content), depth of water in basin and glass cover temperature also play a significant role in the evaporation of water [Muftahet al., 2014].

A lumped system based thermal model is widely used for evaluating the performance of a solar distillation system [Elangoet al., 2015]. This transient mathematical model is derived by employing the energy conservation equation to the water body and the glass cover. These equations involve various heat transfer coefficient namely radiative heat transfer coefficient, convective heat transfer coefficient and evaporative heat transfer coefficient between water, glass cover and ambient air. It is also well established, that evaporative heat transfer coefficient between water and glass coveris a function of respective convective heat transfer coefficient [Tiwari and Sahota, 2017]. Therefore, different researchers have developed different models to estimate the convective heat transfer coefficient for various design and operating conditions. Among these models, Dunkle's model is widely accepted for lower operating temperatures, while for high operating temperatures, model proposed by [Kumar and Tiwari, 1996] is believed to be more accurate.

It is a well-known fact that the water depth in the basin affects the productivity of the solar distillation system [Tiwari and Tiwari, 2006]. There are various studies which confirm that with the increase in depth of water, productivity of the system decreases. Khalifa and Hamood [2009] collected the data of productivity and corresponding water depth, cited in various studies and developed an empirical correlation for the productivity in terms of water depth for a given set of operating conditions. The authors also conducted experimental study and further improved the proposed correlation.

Present study considers a solar still like configuration and proposes an expression for the convective heat transfer coefficient, based on the Kumar and Tiwari[1996] model. This expression, explicitly includes the effect of water depth on the convective heat transfer coefficient. The unknown parameters of the proposed expression are calculated using the inverse heat transfer framework. This framework involves a recursive solution of the forward model which leads to high computational cost. Therefore, a surrogate model is developed using artificial neural networks (ANN) available in MATLAB to reduce the computational cost. In the absence of experiments, the temporally varying temperatures obtained from the solution of an ab initio model is considered as the measurements. Further, the pertinent parameters of the correlation are estimated by minimizing the difference between simulated temperatures obtained from the surrogate

\footnotetext{
* Corresponding Author: :
} 
model and those obtained from the ab initio model, in a least square sense using an evolutionary optimization algorithm namely Genetic Algorithm.

\section{Problem Description}

A cavity with square cross-section $(50 \mathrm{~mm} \times 50 \mathrm{~mm})$, partially filled with water up to $20 \mathrm{~mm}$, is considered for the study (Fig. 1). The cavity is thermally insulated from all sides except the top surface, which is maintained at a constant temperature $(303 \mathrm{~K})$. The water inside the cavity is initially at $323 \mathrm{~K}$, while the air is assumed to be at ambient temperature $(303 \mathrm{~K})$. As the water evaporates, water cools down due to absorption of the latent heat of vaporization which causes gravitational instability in the water and develops weak convective currents in it. As the evaporation continues, the air inside the cavity becomes saturated with water vapour and the temperature of water drops significantly. Since the evaporation of water is a strong function of its temperature and of partial vapour pressure inside the cavity, therefore the evaporation rate of water also decreases. This drop in the evaporation rate suppresses the convection currents and diffusive heat transfer becomes dominant inside the cavity. With this understanding, a one-dimensional, transient, diffusive, simultaneous heat and mass (moisture) transfer model is developed to replace the conventional lumned svstem based heat transfer model.

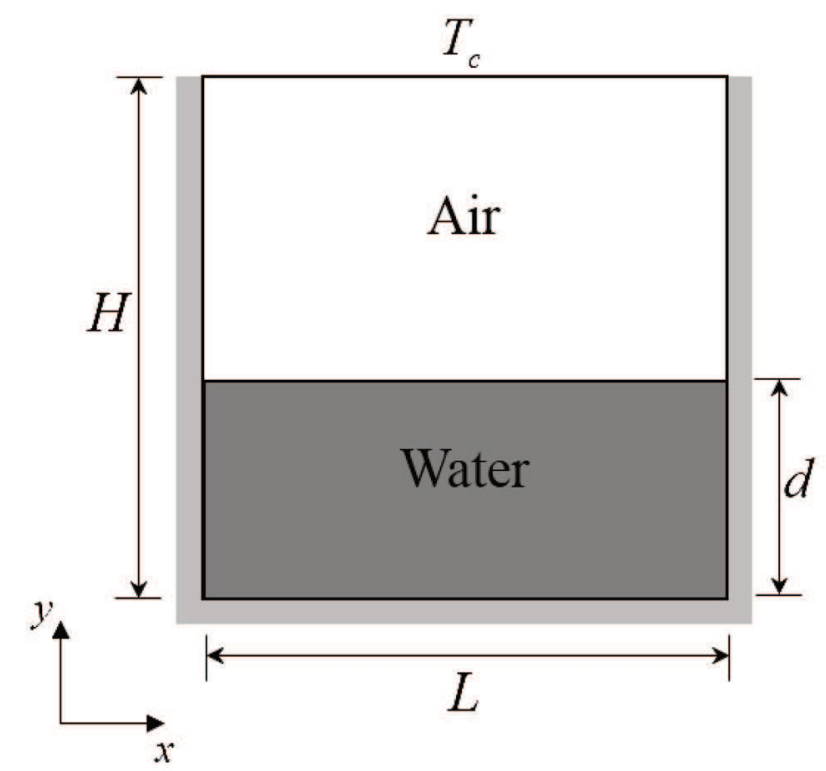

Figure 1: Schematic drawing of the test section isulated from all sides except the top surface, which is maintained at ambient temperature.

\section{Mathematical Modelling}

\subsection{The ab initio model}

Temporally varying transport of heat in one dimension in both air and water can be modelled using the following equation:

$$
\frac{\partial T}{\partial t}=\alpha \frac{\partial^{2} T}{\partial y^{2}}
$$

The bottom surface of the cavity is insulated i.e. $\Delta . T=0$ while the top surface is assumed to be at a constant temperature i.e. $T=303 \mathrm{~K}$. The interfacial boundary conditions for heat transport equations is

$$
-\left.k_{w} \frac{\partial T}{\partial y}\right|_{i}=-\left.k_{a} \frac{\partial T}{\partial y}\right|_{i}+q_{e}
$$

In the above, both air and water are coupled by a diffusive evaporative heat flux. This flux is calculated using the Fick's law of diffusion. Therefore, the interfacial evaporative heat flux can be expressed as:

$$
q_{e}=-\left.\rho_{a} D(T) \frac{\partial \omega}{\partial y}\right|_{i} h_{f g}
$$

The moisture transport takes place in air only, therefore transient onedimensional moisture transport in air can be expressed as

$$
\frac{\partial \omega}{\partial t}=\frac{\partial}{\partial y}\left(D(T) \frac{\partial \omega}{\partial y}\right)
$$

The mass diffusivity of water vapour in air is a strong function of temperature, which is derived from the kinetic theory of ideal gases and expressed as

$$
D=1.87 \times 10^{-10} \frac{T^{2.072}}{p}
$$

Here $p$, is the total pressure of the system which is taken to be 1atm. The air adjacent to the water domain is considered to be saturated with water vapour, therefore the moisture content at the air-water interface is given by

$$
\omega_{s}=0.622 \times \frac{p_{s}}{p-p_{s}}
$$

The saturation pressure of water at a given temperature calculated as:

$$
p_{s}=611.85 \times \exp \left(17.502 \times \frac{T-273.15}{T-32.25}\right)
$$

Similarly, the air near the cold surface is also assumed to be saturated at the cold surface temperature. The ab initio model is solved numerically, using an in-house $\mathrm{C}++$ code to obtain the temperature distribution in the water domain for different time instances. The temperatures obtained from the temporal solution of the $a b$ initio model are utilized as simulated experimental measurements.

\subsection{The Forward model}

The forward model considered here differs from the ab initio model by the fact that, transport of moisture in air is not exclusively modelled in it. Instead, air and water are thermally coupled through evaporative heat flux based on empirical correlations of convective and evaporative heat transfer coefficients. The governing equation for heat transport and corresponding boundary conditions are the same as the $a b$ initio model except the expression for evaporative heat flux used at the air-water interface. Here, the evaporative heat flux is calculated as

$$
q_{e}=h_{e}\left(T_{i}-T_{c}\right)
$$

The evaporative heat transfer coefficient is expressed as [Tiwari and Sahota, 20171

$$
h_{e}=\left(\frac{h_{f g}}{c_{p a}}\right)\left(\frac{M_{w}}{M_{a}}\right)\left(\frac{p}{\left(p-p_{s, i}\right)\left(p-p_{s, c}\right)}\right) h_{c}
$$

And, the convective heat transfer coefficient is expressed as [Kumar and Tiwari, 1996]

$$
h_{c}=N u \frac{k_{a}}{(H-d)}=\frac{k_{a}}{(H-d)} A\left(\frac{d}{H}\right)^{-B}\left(G r^{*} P r\right)^{n}
$$

$A, B$ and $n$ are the unknown parameters, which are estimated using the inverse framework. A modified Grashof number is used in the calculations, which is expressed as:

$$
G r^{*}=\frac{(H-d)^{3} \rho_{s}^{2} g}{\mu_{s}}\left(\frac{M_{s, c} T_{i}}{M_{s, i} T_{c}}-1\right)
$$

An in-house $\mathrm{C}++$ code is developed to solve the forward model numerically, wherein the equations are discretized using Crank-Nicolson finite-difference scheme. A uniform grid is used with $\Delta x=0.1 \mathrm{~mm}$, while time step is chosen to be $\Delta t=0.01 \mathrm{~s}$, after performing the grid-independence and time step independence studies, respectively.Three different grid sizes $\Delta x=0.25 \mathrm{~mm}, 0.125 \mathrm{~mm}$ and $0.1 \mathrm{~mm}$ are considered for grid independence study, while $\Delta \mathrm{t}=0.1 \mathrm{~s}, 0.05$ sand $0.01 \mathrm{~s}$, are taken for the time step independent study as shown in figure 2(a) and 2(b) respectively. 

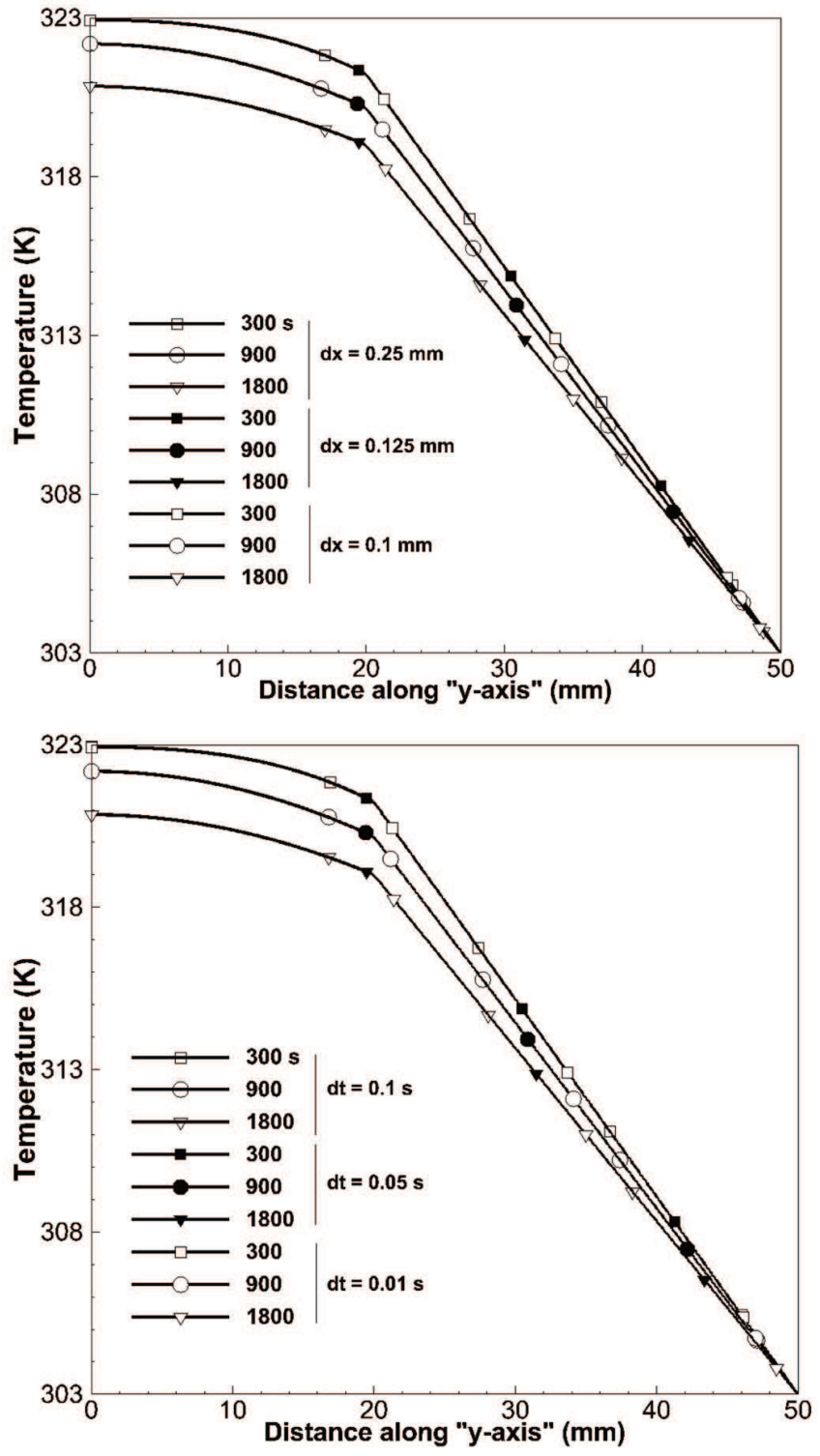

Figure 2: The centreline temperature profile along the y-axis at three time instants $300 \mathrm{~s}, 900 \mathrm{~s}$ and $1800 \mathrm{~s}$

a) Grid independence study using $\Delta x=0.25 \mathrm{~mm}, 0.125 \mathrm{~mm}$ and $0.1 \mathrm{~mm}$

b) Time step independence studyusing $\Delta t=0.1 \mathrm{~s}, 0.05 \mathrm{~s}$ and $0.01 \mathrm{~s}$.

\subsection{Sensitivity Analysis}

Inverse problems concern the determination of "causes" from the information available about the "effects". In general, these are mathematically ill-posed in nature because of the fact that multiple "causes" can lead to the same "effect". Apart from that, these problems are very sensitive to random errors associated with the measurements which are used as the measured input data [Ozisik, 2018]. forward problem simulations can provide information on the best possible ways to carry out the measurements so that the measured data is extremely sensitive to the parameters of interest. Therefore, a sensitivity analysis is performed to obtain this information and reduce the ill-posedness of the problem at hand. In the present case, the sensitivity coefficient is a measure of the variation in temperature due to small changes in the parameters of interest namely $A, B$ and $n$ which appear in the correlation for quantifying the evaporative heat flux at the interface. It is calculated as follows:

$$
S_{P_{i}}=P_{i} \frac{\partial T}{\partial P_{i}}
$$

Temporal variations of sensitivity coefficients are plotted along the height of the cavity, as shown in Fig. 3 (a-c). It is quite clear from the plots that the values of sensitivity coefficient are maximum near the interface. This implies that the temperature in the region near the air-water interface
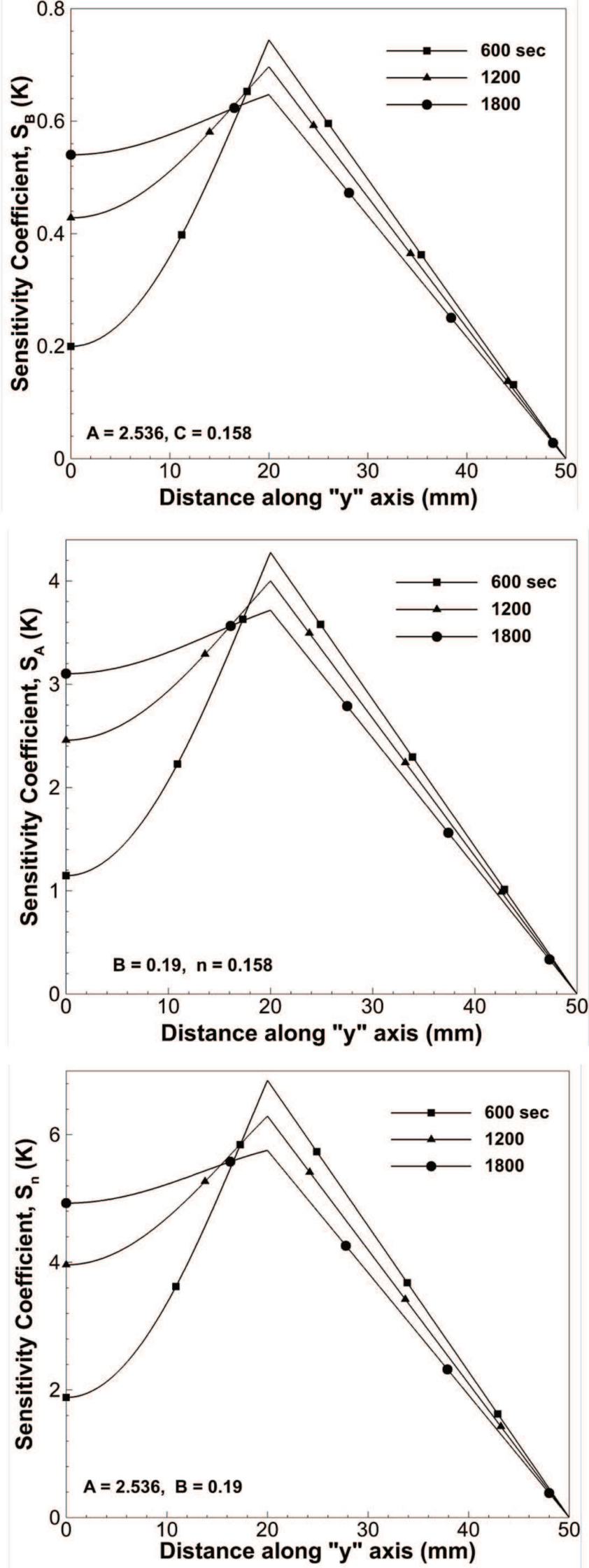

Figure 3: Variation in sensitivity coefficients along the " $y$ " axis for three different time instances i.e. $600 \mathrm{~s}, 1200 \mathrm{~s}$ and $1800 \mathrm{~s}$.

a) Sensitivity coefficient of parameter $A$

b) Sensitivity coefficient of parameter $B$

c) Sensitivity coefficient of parameter $n$ 
changes very rapidly and is a strong function of the evaporative heat flux at the interface. Therefore, five different locations at an interval of $0.5 \mathrm{~mm}$ in the water domain beneath the air-water interface are selected. Since the sensitivity coefficients increase with time, therefore six different instances at an interval of $300 \mathrm{~s}$ till $1800 \mathrm{~s}$ are selected as well. A combination of the above selected spatial and temporal coordinates is used to carry out the temperature measurements. It is worth mentioning here that, in the event of absence of measured temperature data, temperatures obtained at the above mentioned coordinates are used as simulated measurements for carrying out the estimation of the pertinent parameters.

\section{$3.4 \quad$ Fast forward model}

In an inverse heat transfer problem, the forward model is solved recursively to estimate the values of unknown parameters by minimizing the objective function. In this study, a computationally economic and fast surrogate to the original forward model is developed using artificial neural networks (ANN) to reduce the computational cost considerably. The ANN is a machine learning tool, which mimics the mechanism of human brain. It is constructed with various nodes which are connected through various connection weights, such as brain's neurons are connected through the various neural connections [Kim, 2017]. A network is modelled in different layers, wherein the input parameters are puts in input layer, the desired output parameters are placed in output layers. These two layers are connected through one or more layers known as hidden layers. The connections between these layers comprises various weights and biases which also stored theinformation during the training (learning) of the network. The training involves a systematic modification in the weights and biases in order to reduce the error between the input layer and the output layer. The errors of hidden layers are determined using the backpropagation algorithmin which the output error propagates from the output layer to the input layer. The back-propagation algorithm also involves the adjustment of weights and biases of connecting nodes.

Standard neural network module available in MATLAB is employed to develop a feed forward back propagation based ANN. Parameters of interest namely $\mathrm{A}, \mathrm{B}$ and $n$ in a prescribed range obtained from literature on solar distillation systemsand physical time are used as inputs and corresponding temperatures at pre-determined locations obtained by solving the forward problem using the prescribed values of A, B and $n$ are used as outputs of the ANN.

The prescribed values of $A, B$ and $n$ are taken as 2.536 [Tiwari and Tiwari, 1996], 0.19 [Khalifa and Hamood, 2009] and 0.158 [Tiwari and Tiwari, 1996] respectively. In order to construct the ANN based surrogate model, an input data set comprising of 200 random values of $t, A, B$ and $n$ is generated, in which the time $t$, varies from 0 to $1800 \mathrm{~s}$ while the range of other parameters are taken as $\pm 99 \%$ of the reference values. The forward model is solved for these 200 sets to generate the corresponding temperature distribution in water. As discussed above, temperatures at 5 pre-determined spatial locations in water beneath the air-water interface at an interval of $0.5 \mathrm{~mm}$ for 6 time instances at an interval of $300 \mathrm{~s}$ up to $1800 \mathrm{~s}$, obtained by solving the forward model for the prescribed input data sets are considered as the corresponding output data sets.

Out of considered 200 input-output data sets, 150 data sets are used to train the network. The feed forward back propagation algorithm is used with a single hidden layer in the network. Bayesian regularization is used as the training algorithm with mean square error (MSE) and correlation coefficient $\left(\mathrm{R}^{2}\right)$ as the performance indices. The number of neurons in the hidden layer are determined by the neuron independence test [Chandaet al., 2018], as reported in table 1 . The remaining 50 data sets are not involved in the training and only used after development the network. These data sets are used for the validation of the developed network. This fast forward model (surrogate) is used to replace the forward model in the inverse framework during the minimization of the objective function.

\subsection{Inverse heat transfer framework}

In order to estimate the unknown parameters, the forward model is solved for different assumed values of $A, B$ and $n$. Temperatures at predetermined locations and time instants as mentioned in the previous section, obtained by solving the forward problem are considered as simulated measurements. The parameters of interest namely A, B and $n$ are obtained by minimising the difference between the measured temperatures and those obtained from the ab-initio model, in a least square sense. Based on the above discussion, the objective function is expressed as follows:

$$
e=\sum_{i=1}^{6} \sum_{i=1}^{5}\left[\left(T_{\text {exp }, i}-T_{\text {sim }, i}\right)^{2}\right]
$$

The above function is minimized using Genetic algorithm to estimate the parameters of interest namely $A, B$ and $n$. It is a non-traditional optimisation technique which derives inspiration from the process of natural selection, evolution and genetics. Stochastic nature of the algorithm as well as its ability to circumvent gradient calculation of the objective function makes it an attractive choice. The algorithm starts from a pool of individual values of parameters of interest ( $A, B$ and $n$ ) which are likely candidate solutions to the minimisation problem. Operations such as selection, mating, crossover, mutation and elitism are performed on the initial population. It is expected that with progress in the iterations better solutions of parameters of interest are obtained which would inevitably minimize the objective function at hand[Goldberg, 1989].

\section{Results and Discussion}

\subsection{Estimation of parameters using exact synthetic data}

As mentioned earlier, the enclosure is partially filled with heated water maintained at $323 \mathrm{~K}$, up to $20 \mathrm{~mm}$ and the top surface of the enclosure is maintained at $303 \mathrm{~K}$, keeping all other sides thermally insulated. The parameters of convective heat transfer coefficient (eq. 10) are estimated by minimizing the objective function (eq. 12) using genetic algorithm. The search range for estimating the parameters is limited to $\pm 90 \%$ of the corresponding prescribed values as taken from literature. The values of parameters $A, B$ and $n$ retrieved for exact synthetic data is reported in table 2 .

\subsection{Estimation of parameters using noisy data}

After estimating the parameters using exact synthetic data, noisy synthetic data is generated by sprinkling random noise to the temperatures obtained from the $a b$ initiomodel. The sprinkled noise has a Gaussian distribution with zero mean and $\pm 0.5 \mathrm{~K}$ standard deviation. 100 sets of synthetic data are generated and for these noisy values of temperature the parameters are re-estimated. The mean values of the re-estimated parameters along with respective standard deviations are reported in table 2 .

Table 2: Retrieved values of pertinent parameters

\begin{tabular}{c|c|c|c}
\hline Parameters & $\begin{array}{c}\text { Reference } \\
\text { values }\end{array}$ & $\begin{array}{c}\text { Retrieved values } \\
\text { (No noise) }\end{array}$ & $\begin{array}{c}\text { Retrieved values } \\
\text { (with noise) }\end{array}$ \\
\hline$A$ & 2.536 & 0.293 & $0.261 \pm 0.111$ \\
$B$ & 0.19 & 0.147 & $0.170 \pm 0.133$ \\
$n$ & 0.158 & 0.126 & $0.142 \pm 0.047$ \\
\hline
\end{tabular}

The initial search range of the parameters is very large, therefore the standard deviations of these parameters are very high. Therefore, a narrow search range has been chosen based on the parameters obtained through initial search range. This modified search range is $\mu \pm 0.5 o$, where $\mu$ and $o ́$ are the mean and standard deviation of the corresponding parameter. The parameters evaluate using this modified search are reported in table 3 . It is quite clear form the table 3 , that the mean values of parameters did not change significantly while the standard deviations reduced drastically. The parameters obtained using the synthetic data for both search ranges (initial and modified) are also fitted into the probability distribution function (PDF) and plotted in the figure 4(a-c) for $A, B$ and $n$ respectively.

The parameters obtained using the modified search range are used to solve the forward model for initial water temperature, $T_{i}=323 \mathrm{~K}$, and water depth, $d=20 \mathrm{~mm}$. The temperature distribution obtained from the forward model is compared with the temperature distribution obtained from the $a b$ initio model, as shown in figure 5. The maximum error observed is $0.15 \mathrm{~K}$.

\section{Conclusions}

A methodology is developed to estimate the interfacial evaporative heat flux using an inverse heat transfer approach. The pertinent parameters are estimated by minimizing a least square based objective function, in which the solution of $a b$ initio model is employed as the measured temperature. A surrogate model is developed to replace the computationally expensive forward model to obtain the simulated temperature data. Artificial neural networks and genetic algorithms are utilized for surrogate modelling and minimization of objective function, respectively. The parameters are estimated for both exact and noisy synthetic temperature data. The temperature distribution obtained by solving the forward model using the estimated parameters show a good match with the temperature distribution obtained from $a b$ initio model, thereby establishing the efficacy of the developed methodology. 

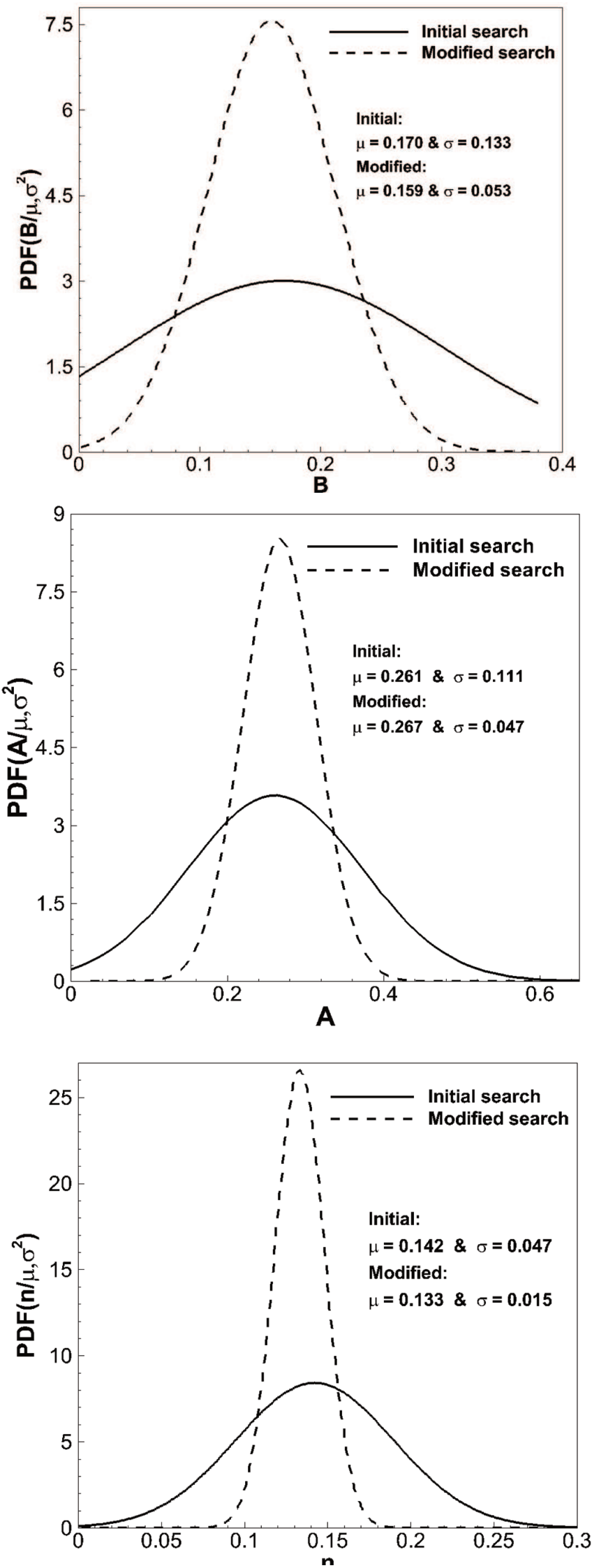

Figure 4: Probability distribution functionof the estimated parameters from synthetic data generated by sprinkling the Gaussian noise a) PDF of the estimated values of parameters $A$

b) PDF of the estimated values of parameters $B$

c) PDF of the estimated values of parameters $n$

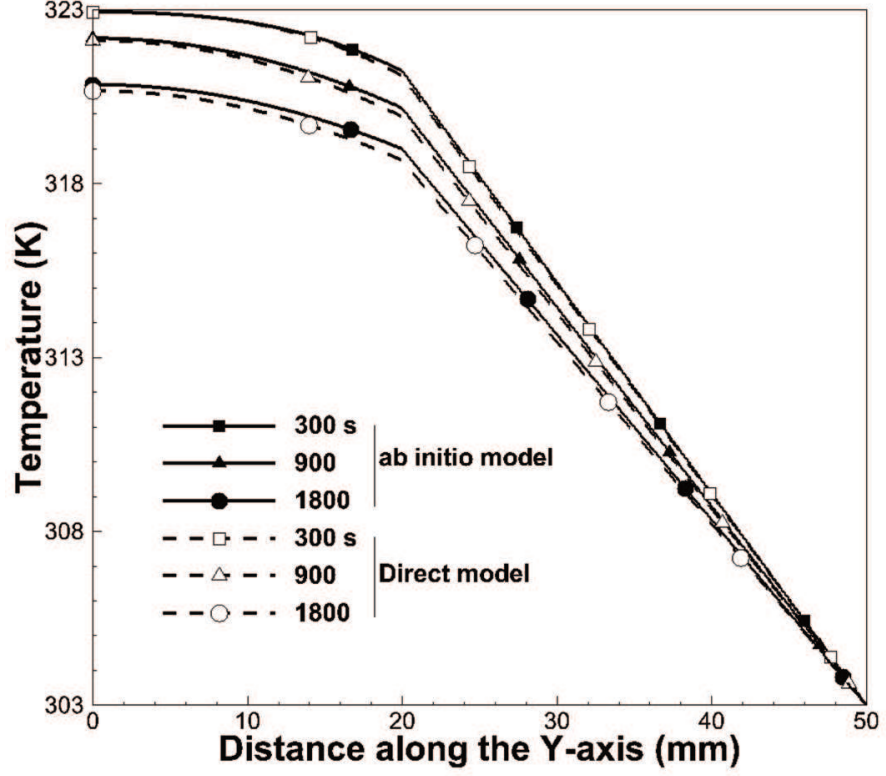

Figure 5: Comparison of temperature profile along the height of test cell for $a b$ initio model and direct based on estimated values of $A, B$ and $n$.

\section{Nomenclature}

$A, B, n$ Unknown parameters

$c_{p a} \quad$ Sp. Heat capacity of water at constant pressure, $\mathrm{J} / \mathrm{kg}-\mathrm{k}$

$d^{p a} \quad$ Binary diffusion coefficient of water-vapour and air, $\mathrm{m}^{2} / \mathrm{s}$

$D \quad$ Water depth in enclosure, $\mathrm{m}$

$e \quad$ Objective function/error

$G r^{*} \quad$ Modified Grashof number

$H \quad$ Height of enclosure, $\mathrm{m}$

$h$. Convective heat transfer coefficient, $\mathrm{W} / \mathrm{m}^{2} \mathrm{k}$

$h$ Evaporative heat transfer coefficient, $\mathrm{W} / \mathrm{m}^{2} \mathrm{k}$

$h_{f g}^{e} \quad$ Latent heat of water, J/kg

$i^{18} \quad$ Subscript for interface

$k_{a} \quad$ Thermal conductivity of air, $\mathrm{W} / \mathrm{m}-\mathrm{K}$

$k_{w}^{a} \quad$ Thermal conductivity of water, $\mathrm{W} / \mathrm{m}-\mathrm{K}$

$L^{w} \quad$ Width of test cell, $\mathrm{m}$

$M_{a} \quad$ Molecular mass of air, $\mathrm{mol} / \mathrm{g}$

$M^{a} \quad$ Molecular mass of saturated air at glass cover/cold surface, $\mathrm{mol} / \mathrm{g}$

$M_{s, i}^{s, c} \quad$ Molecular mass of saturated air adjacent to interface, mol/g

$M \quad$ Molecular mass of water, $\mathrm{mol} / \mathrm{g}$

$\mathrm{Nu} \quad$ Nusselt number

$P \quad$ Total pressure, $\mathrm{N} / \mathrm{m}^{2}$

Pr Prandlt number

$p$ saturation pressure at a specific temperature, $\mathrm{N} / \mathrm{m}^{2}$

$p \quad$ Saturation pressure of water at the air-water interface, $\mathrm{N} / \mathrm{m}^{2}$

$p_{s, i} \quad$ Saturation pressure of water at glass cover/cold surface, $\mathrm{N} / \mathrm{m}^{2}$

$q \quad$ Interfacial evaporative heat flux, $\mathrm{W} / \mathrm{m}^{2}$

$S_{P} \quad$ Sensitivity coefficient of parameter $P, \mathrm{~K}$

$t \quad$ Temperature, $\mathrm{K}$

$T \quad$ Time, s

$T_{c} \quad$ Temperature of glass cover/cold surface, $\mathrm{K}$

$T$. Temperature of air-water interface, $\mathrm{K}$

$\dot{a}^{i} \quad$ Thermal diffusivity, $\mathrm{m}^{2} / \mathrm{s}$

$\tilde{n} \quad$ Density of air, $\mathrm{kg} / \mathrm{m}^{3}$

$\tilde{n}_{\text {s }} \quad$ Density of saturated air, $\mathrm{kg} / \mathrm{m}^{3}$

$\grave{u}^{s} \quad$ Sp. Humidity of air

$\grave{u}_{s} \quad$ Sp. Humidity of saturated air

$\mu_{a} \quad$ Dynamic viscosity of air, Pa.s

$\mu_{s} \quad$ Dynamic viscosity of saturated air, Pa.s

\section{References}

[1] Chanda S, Muralidhar K, Nimdeo YM, 2018, Joint estimation of thermal and mas diffusivities of a solute-solvent system using ANN-GA based inverse framework. International Journal of Thermal Sciences, 123, 27-41.

[2] Elango C, Gunasekaran N, Sampathkumar K, 2015, Thermal models of solar stillA comprehensive review, Renewable and Sustainable Energy Reviews, 47, 856911.

[3] Goldberg D, 1989, Genetic algorithms in search, optimization and machine learning. New York: Addison-Wesley Publishing Company, Inc. 
[4] Khalifa AJN, Hamood AM, 2009, On the verification of the effect of water depth on the performance of basin type solar stills, Solar Energy, 83(8), 1312-1321.

[5] Kim P, 2017, Matlab deep learning. With Machine Learning, Neural Networks and Artificial Intelligence, 130.

[6] Kumar S, Tiwari GN, 1996, Performance evaluation of an active solar distillation system, Energy, 21(9), 805-808.

[7] Muftah AF, Alghoul MA, Fudholi A, Abdul-Majeed M M, Sopian K, 2014, Factors affecting basin type solar still productivity: A detailed review, Renewable and Sustainable Energy Reviews, 32, 430-447.
[8] Ozisik MN, 2018, Inverse heat transfer: fundamentals and applications, Routledge.

[9] Rajaseenivasan T, Prakash R, Vijayakumar K, Srithar K, 2017, Mathematical and experimental investigation on the influence of basin height variation and stirring of water by solar PV panels in solar still, Desalination, 415, 67-75.

[10] Tiwari AK, Tiwari GN, 2006, Effect of water depths on heat and mass transfer in a passive solar still: in summer climatic condition, Desalination, 195(1-3), 78-94.

[11] Tiwari GN, Sahota L, 2017, Advanced Solar-Distillation Systems: Basic Principles, Thermal Modelling, and Its Application, Springer. 